\title{
Patterns of Expression of Vascular Endothelial Growth Factor (VEGF) and VEGF Receptors in Mice Suggest a Role in Hormonally Regulated Angiogenesis
}

\author{
Dorit Shweiki, Ahuva Itin, Gera Neufeld, ${ }^{\star}$ Hela Gitay-Goren, ${ }^{\star}$ and Eli Keshet \\ Department of Molecular Biology, The Hebrew University-Hadassah Medical School, Jerusalem 91010; \\ and ${ }^{*}$ Department of Biology, Technion, Haifa 32000, Israel
}

\begin{abstract}
Vascular endothelial growth factor (VEGF) is a secreted endothelial cell-specific mitogen. To evaluate whether VEGF may play a role in angiogenesis, we have determined the spatial and temporal patterns of expression of VEGF and VEGF receptors during natural angiogenic processes taking place within the female reproductive system. Four angiogenic processes were analyzed: neovascularization of ovarian follicles, neovascularization of the corpus luteum, repair of endometrial vessels, and angiogenesis in embryonic implantation sites. During all processes, VEGF mRNA was found to be expressed in cells surrounding the expanding vasculature. VEGF was predominantly produced in tissues that acquire new capillary networks (theca layers, lutein cells, endometrial stroma, and the maternal decidua, respectively). VEGF-binding activity, on the other hand, was found on endothelial cells of both quiescent and proliferating blood vessels. These findings are consistent with a role for VEGF in the targeting of angiogenic responses to specific areas. Using in situ hybridization, we show that VEGF is expressed in 10 different steroidogenic and /or steroid-responsive cell types (theca, cumulus, granulosa, lutein, oviductal epithelium, endometrial stroma, decidua, giant trophoblast cells, adrenal cortex, and Leydig cells). Furthermore, in some cells upregulation of VEGF expression is concurrent with the acquisition of steroidogenic activity, and expression in other cell types is restricted to a particular stage of the ovarian cycle. These findings suggest that expression of VEGF is hormonally regulated. We propose that excessive expression of VEGF during gonadotropin-induced ovulation may contribute to the development of ovarian hyperstimulation syndromes by virtue of the vascular permeabilization activity of this factor. (J. Clin. Invest. 1993. 91:2235-2243.) Key words: neovascularization • ovary • steroidogenesis • vascular permeability • in situ hybridization
\end{abstract}

\section{Introduction}

Angiogenesis, the formation of new blood vessels as extensions of existing vessels, is a complex multistep process. Angiogenesis is thought to be initiated by local activation of genes encoding diffusible angiogenic factors (or, alternatively, by release of

Address correspondence to Dr. Eli Keshet, Department of Molecular Biology, The Hebrew University-Hadassah Medical School, Jerusalem 91010, Israel.

Received for publication 7 August 1992 and in revised form 16 December 1992.

J. Clin. Invest.

(c) The American Society for Clinical Investigation, Inc.

0021-9738/93/05/2235/09 \$2.00

Volume 91, May 1993, 2235-2243 preformed factors from their stores). The secreted angiogenic factors, in turn, initiate a cascade of downstream responses, that result in the growth of new capillaries towards the sites emitting the angiogenic signal. Since the turnover rates of vascular endothelial cells are extremely low, angiogenesis is generally a quiescent process in the healthy adult organism. A marked exception is the female reproductive system, where the need for additional vasculature is constantly imposed by the cyclic evolution of transient structures and by the cyclic repair of damaged tissues. Specifically, in every estrous cycle, the sequential development of ovarian follicles and corpora lutea $(\mathrm{CL})^{1}$ is accompanied by concomitant development of elaborate capillary networks. The latter are formed in the periphery of each growing follicle, or in the vicinity of lutein cells, respectively (3). Similarly, in the uterus, blood vessels of the functional endometrium undergo cyclic extensions and repair, compensating for vessels that have been damaged or slought off (4). Extensive neovascularization of the endometrium also takes place upon decidual transformation in response to embryonic implantation. The increase in decidual mass is accompanied by induction of neovascularization and formation of an anastomosing network traversing the maternal decidua and converging on the embryo (5). This vascular network supplies the maternal contribution of the future placenta. These four processes represent independent examples of programmed selflimiting angiogenesis within the female reproductive system.

Studies from many laboratories, using naturally avascular tissues as model systems, have shown that a relatively large number of factors are capable of eliciting an angiogenic response (for reviews, see references 6-8). Yet the identity of factor(s) that initiate neovascularization under natural settings is not known. The reproductive system is an attractive system to critically evaluate the relevance of putative angiogenic factors to natural angiogenesis. It has the advantages that angiogenesis can be readily induced and synchronized by gonadotropins, and that it occurs over a relatively short period of time in a well-characterized schedule. Furthermore, vascular extension follows known routes with respect to the vessels of origin and the target tissue (9). Thus, specimens can be retrieved at successive stages of the ongoing process. Elucidating spatial and temporal patterns of expression for each candidate angiogenic growth factor, in conjunction with the pattern of expression of its cognate receptors, defines the framework of its potential in vivo action.

Vascular endothelial growth factor (VEGF), is a heparinbinding, secreted dimeric glycoprotein (for review, see reference 10). VEGF display an angiogenic activity in the cornea

1. Abbreviations used in this paper: CL, corpora lutea; OHSS, ovarian hyperstimulation syndrome; VEGF, vascular endothelial growth factor. 

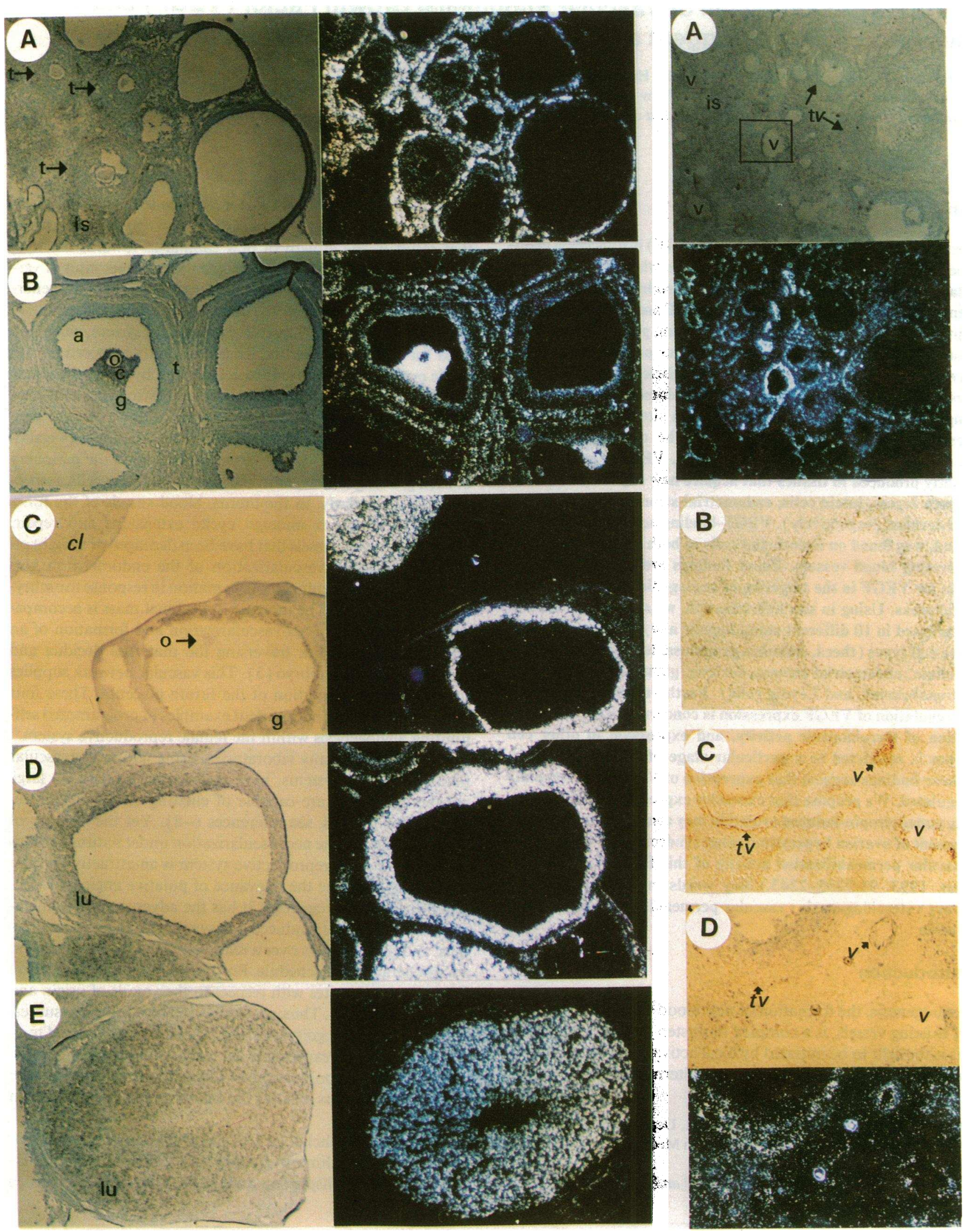
and chorioallantoic assays (11-13), induces the migration of monocytes in vitro (14), and is a mitogen with a target cell specificity that seems to be restricted to vascular endothelial cells $(15,16)$. Recent in situ analysis by Phillips et al. (17) have shown that VEGF-binding activity is expressed on endothelial cells comprising the vascular network of the corpus luteum, while the ligand is produced by the lutein cells (10). These findings prompted us to further investigate whether VEGF may play a role in the angiogenic processes discussed above. Here, we show that in the reproductive tract, VEGF is specifically expressed in the vicinity of proliferating endothelium, in a pattern that may provide directionality to the extending vasculature. VEGF receptors, on the other hand, are constitutively expressed in the endothelium, regardless of its proliferative status.

It is most likely that the cyclic angiogenic waves in the female reproductive system are coordinated by hormones. It is assumed, therefore, that these processes are mediated by an angiogenic factors that are, either directly or indirectly, hormone inducible. To examine whether expression of VEGF is regulated by hormones under physiological conditions, we extended the in situ analysis to steroidogenic cells outside the female reproductive system. Interestingly, all cell types shown here to express VEGF are steroidogenic and/or steroid-responsive cells.

\section{Methods}

Animals, organs, and tissues. Hyperstimulation of ovaries was induced in immature (25-d-old) female rats by injecting $25 \mathrm{IU}$ of pregnant mare's serum gonadotropin (G-4877; Sigma Immunochemicals, St. Louis, MO). 5 IU of human chorionic gonadotropin (GG-2; Sigma Immunochemicals) was injected $46 \mathrm{~h}$ later. Whole reproductive tracts (including ovaries, oviducts, and uteri) were withdrawn at the indicated times, representing successive stages in the ovarian cycle.

For analysis of embryonic implantation sites, 8-w-old C57BL/6 female mice were hyperstimulated and mated with C57BL/ 6 males. Embryos were staged as $0.5-\mathrm{d}$ postcoitum at noon of the day in which the vaginal plug was observed. Whole implantation sites (including maternal, extraembryonic, and embryonic tissues) were collected.

Pseudopregnancy was induced in mice by injecting gonadotropins, mating with a vasectomized male, and intrauterine injection of oil at $3.5 \mathrm{~d}$ postcoitum (18).

Tissues were fixed overnight in $4 \%$ paraformaldehyde in PBS. Fixed specimens were then incubated in $0.5 \mathrm{M}$ sucrose in PBS before embedding in Tissue-Tek OCT embedding medium (Miles Inc., Kanakee, IL) and in situ hybridization.
In situ hybridization. This procedure was performed essentially as described by Hogan et al. (19). In brief, 10-mm thick frozen sections were collected on poly (L-lysine)-coated glass slides, refixed, and dehydrated in graded ethanol solutions. Before hybridization, sections were pretreated successively with $0.2 \mathrm{M} \mathrm{HCl}$, pronase $(0.125 \mathrm{mg} / \mathrm{ml}), 4 \%$ paraformaldehyde, and acetic anhydride in triethanolamine buffer. Hybridization was carried out at $50^{\circ} \mathrm{C}$ overnight in a solution containing $50 \%$ ( $\mathrm{vol} / \mathrm{vol}$ ). formamide $/ 0.3 \mathrm{M} \mathrm{NaCl}$ and ${ }^{35} \mathrm{~S}$-labeled RNA probe ( 2 $\times 10^{8} \mathrm{cpm} / \mathrm{ml}$ ). Washing was performed under stringent conditions that included an incubation at $50^{\circ} \mathrm{C}$ for $>14 \mathrm{~h}$ in $50 \%$ formamide $/ 0.3$ $\mathrm{M} \mathrm{NaCl}$ and a $30-\mathrm{min}$ incubation at $37^{\circ} \mathrm{C}$ with $\mathrm{RNase} A(20 \mathrm{mg} / \mathrm{ml})$. Autoradiography was performed using Kodak NTB-2 nuclear track emulsion with exposure for 5-9 d. In the initial experiments, control hybridizations with a riboprobe in the "sense" orientation were also carried out.

Hybridization probes. The following DNA fragments were cloned onto the polylinker of a PBS vector (Stratagene, La Jolla, CA) to serve as templates for synthesis of specific cRNAs: A 1.8-kb-long cDNA fragment containing $\sim 3^{\prime}$ two thirds of the coding region, as well as the entire 3'-untranslated region of mouse VEGF; an 8.8-kb-long EcoRISalI fragment derived from a human von Willebrand factor cDNA clone (20).

Constructs in PBS vector were linearized by digestion with the appropriate restriction endonuclease to allow synthesis of an ${ }^{35} \mathrm{~S}$-labeled complementary RNA in either the antisense or sense orientation (using T3 or T7 RNA polymerase). RNA probes were fragmented by a mild alkaline treatment before use for in situ hybridization.

In situ VEGF-binding analysis. Recombinant human VEGF ( 165 amino-acid long form) was produced in SF9 cells infected with a baculovirus containing a cloned VEGF cDNA under control of the polyhedrin promoter. Production and purification of the factor were performed as previously described (21), with the addition of a cationexchange chromatography step. VEGF was iodinated by the chloramine-T method as described by Vaismann et al. (22).

Binding of $\left[{ }^{125} \mathrm{I}\right]$ VEGF to frozen sections was performed as described by Marchetti et al. (23), with some modifications. Briefly, organs were incubated overnight in $0.5 \mathrm{M}$ sucrose in PBS before embedding in Tissue-Tek OCT embedding medium. 10-mm thick frozen sections were collected on gelatine-coated glass slides. After preincubation (PBS, $30 \mathrm{~min}$, room temperature), slides were incubated ( $1 \mathrm{~h}$, room temperature) in PBS solution containing $5 \mathrm{ng} / \mathrm{ml}$ of $\left[{ }^{125} \mathrm{I}\right]$ VEGF (specific activity: $150,000 \mathrm{cpm} / \mathrm{ng}$ ), $1 \mathrm{mg} / \mathrm{ml}$ heparine, and $0.2 \%$ gelatine. Slides were subsequently washed with ice-cold PBS and, briefly (1 $\mathrm{min}$ ), with water. Sections were then fixed for $10 \mathrm{~min}$ in $2.5 \%$ glutaraldehyde solution in $50 \mathrm{mM}$ phosphate buffer ( $\mathrm{pH} 6.5$ ), washed in phosphate buffer, air dried, and autoradiographed using Kodak NTB-2 nuclear track emulsion. Exposure time was 1-2 d.

Immunocytochemical staining. Sections were stained with a polyclonal rabbit anti-human von Willebrand factor ( 1:200) (Dako Corp., Carpinteria, CA) for $90 \mathrm{~min}$ at room temperature. The primary anti-

Figure 1. Expression of VEGF mRNA during development of ovarian follicles and corpus luteum. In situ hybridization with a VEGF-specific probe. Left and right figures show the same section photographed under bright field and dark field illumination, respectively. $(A)$ Early stages in follicular development. Arrows point at theca layers of preantral follicles and follicles with a small antrum. $(B)$ Developed follicles showing high level of expression in cumulus cells. $(C)$ Immediate preovulatory follicle. $(D)$ An early stage in CL development. $(E)$ Fully developed CL. $v$, blood vessels; $i$, ovarian interstitial tissue; $t$, theca; $o$, oocyte; $c$, cumulus; $g$, granulosa; $a$, antrum; $c l$, corpus luteum; $l u$, lutein cells.

Figure 2. [ ${ }^{125}$ I]VEGF binding sites within the ovary. Ovary sections, representing growing follicles, were assayed for [ $\left.{ }^{125} \mathrm{I}\right]$ VEGF binding as described in Methods. $(A)$ A section showing multiple transverse sections through large medullar vessels $(v)$, interstitial tissue $(i s)$, and a number of growing follicles. Bright field (top) and dark field (bottom) photographs. Labeling is mostly in large vessels and in the periphery of follicles. $(B)$ A larger magnification of the area boxed in $A$. Note that autoradiographic grains are detectable primarily on the endothelial lining of the blood vessel. ( $C$ and $D$ ) Colocalization of von Willebrand factor immunoreactivity and [ ${ }^{125}$ I]-VEGF binding sites. $(C)$ Immunostaining with the endothelial cell-specific von Willebrand factor (see Methods for details). Note staining (brown reaction products) on large vessels $(V)$, as well as on capillaries embedded in the theca layers of the follicle $(t v)$. (D) An adjacent serial section analyzed for [ ${ }^{125}$ I] VEGF binding (bright field and dark field photographs). Note colocalization of binding sites with von Willebrand factor immunoreactivity. 
body was detected using the avidin-biotin peroxidase system (Vectastain ABC; Vector Labs, Burlingame, CA) with diaminobenzidine as the chromogen.

\section{Results}

In situ hybridization of sectioned tissues enabled us to determine the sites of VEGF production under natural settings that preserve the correct interactions between different cell types. Organs were retrieved at times where neovascularization of tissues is ongoing, immediately withdrawn into a fixative, and processed for in situ hybridization analysis using VEGF-specific, ${ }^{35} \mathrm{~S}$-labeled antisense ribopobrobes. Mouse VEGF cDNA was cloned in our laboratory from a mouse brain cDNA library. The 1.8-kb-long cDNA fragment used throughout this study contained $\sim 3^{\prime}$ two thirds of the coding region, as well as the entire $3^{\prime}$-untranslated region (the VEGF-specific probe detected only the expected VEGF-containing bands in preliminary genomic DNA blotting experiments [data not shown]). In situ analysis of mRNA was preferred over in situ immunodetection of the encoded protein because the localization of the mRNA unequivocally identifies the producer cells, whereas VEGF is known to be secreted and might also be sequestered elsewhere in the tissue (24). To define potential targets for locally released VEGF, we identified cells expressing functional VEGF receptors by performing in situ ligand binding analysis. To this end, ${ }^{125}$ I-labeled VEGF $_{165}$ (produced in a baculovirus vector system) was used.

Neovascularization of ovarian follicles and corpus luteum. To follow VEGF expression during follicular development, we used follicle-stimulating hormone-primed female rats. In this experimental system, a large cohort of follicles is induced to develop in a roughly synchronous manner (25). Whereas small preantral follicles have no special vascular supply of their own, concomitant with further growth follicles acquire individual vascular sheaths. The follicular networks originates at vessels residing in the medulla, extends towards each growing follicle, and forms two concentric capillary networks embedded in the theca externa and theca interna, respectively. The whole process is completed within $1 \mathrm{~d}$ from the onset of follicular growth. Focusing on preantral follicles and on follicles with small antrum (i.e., follicles undergoing neovascularization), VEGF was expressed in the interstitial tissue of the ovary and in the theca layers (Fig. $1 A$ ). The VEGF produced and secreted by these cells is likely to act on nearby cells, provided that the target cells express the appropriate receptors. Recent work has shown that the vast majority of VEGF binding appears to be associated with endothelial cells, in general, and to correspond to the pattern of ovarian vascularization, in particu$\operatorname{lar}(10,26)$. We have determined the cell populations that bind VEGF at the time of follicular neovascularization by in situ receptor-ligand binding analysis. In agreement with the previous findings, VEGF receptors were detectable on the endothelium of large medullar vessels (from which the thecal networks originate), on cells interspersed in the stroma (presumably endothelial cells), and in capillaries arranged in the peripheral theca layers of growing follicles (Fig. 2). Thus, it appears that the primary target of the ligand, which is produced along the path of capillary extension toward growing follicles, is the endothelium. Furthermore, expression of VEGF in the theca layers (but not in the inner granulosa) suggests that the locally se- creted protein may target capillary extension to the periphery of the follicle.

Concomitantly with further growth and maturation of follicles, the site of VEGF expression shifts to additional ovarian compartments (Fig. 1, $B-E$ ). The first cells inner to the theca to express VEGF are cumulus cells engulfing the oocyte (Fig. 1 $B)$. In the granulosa compartment, high levels of VEGF mRNA are detectable only at the immediate preovulatory stage (Fig. $1 C$ ). Shortly after ovulation, as granulosa cells transform to lutein cells and development of the corpus lutem ensues, the predominant site of VEGF expression is lutein cells (Fig. 1, $D$ and $E$ ). Fig. 1 thus portrays a dynamic pattern of VEGF expression, changing in parallel to the gonadotropin stimuli received by different steroidogenic ovarian cells and in parallel to changes in their steroid outputs.

With respect to angiogenesis, the pattern of VEGF expression shown in Fig. 1 suggests that VEGF may also play a role in the second angiogenic process taking place within the ovary, namely, neovascularization of the corpus luteum. Before ovulation, the thecal capillary network is sufficient to provide the granulosa cells residing only few cell layers away. Upon development of the corpus luteum, however, lutein cells proliferate and gradually fill the entire space of the former antrum. To satisfy the added perfusion requirements, capillaries invade the corpus luteum and form an elaborate network whereby each lutein cell resides in close proximity to blood capillaries. A role for VEGF in the development of the CL vasculature is suggested by the abundant production of VEGF by all lutein cells during the period where concurrent proliferation of lutein cells and endothelial cell takes place (Fig. $1 D$ ). Consistent with its role as a paracrine endothelial cell mitogen, it has been shown that the CL vasculature possesses a VEGF-binding activity (reference 10 and our data not shown). Notably, lutein cells continue to express VEGF in the fully developed, functional $\mathrm{CL}$ (Fig. $1 E$ ). The latter observation confirms the findings of Phillips et al. (17).

Angiogenesis in the endometrium. Throughout the reproductive life span, the endometrium undergoes cyclic changes in its secretory activity and structure, in correlation with the cyclic growth and maturation of ovarian follicles, and under control of ovarian hormones. The endometrial unique system of blood vessels also undergoes cyclic development. Specifically, after the partial destruction of the endometrium at the end of each cycle, the distal portion of the spiral arteries undergoes regeneration. Lengthening and coiling of arteries is under the influence of estrogens and progesterone, beginning at the proliferative phase of the endometrium and continuing during the secretory phase.

To evaluate whether VEGF may play a role in neovascularization of the endometrium, we analyzed its pattern of expression in the reproductive track during different stages of the cycle (Fig. 3). VEGF was found to be expressed in the estrogen-responsive, secretory columnar epithelium, lining both the oviducts (Fig. $3 A$ ) and the uterus (Fig. $3 B$ ). A different pattern of expression was observed, however, when, under the influence of progesterone, maximal development of the secretory endometrium, and concomitant development of blood vessels takes place. Now, the site of VEGF expression shifts to cells of the underlying stroma composing the functional endometrium (note, for comparison, the lack of expression in the peripheral myometrium) (Fig. $3 \mathrm{C}$ ). These observations, 

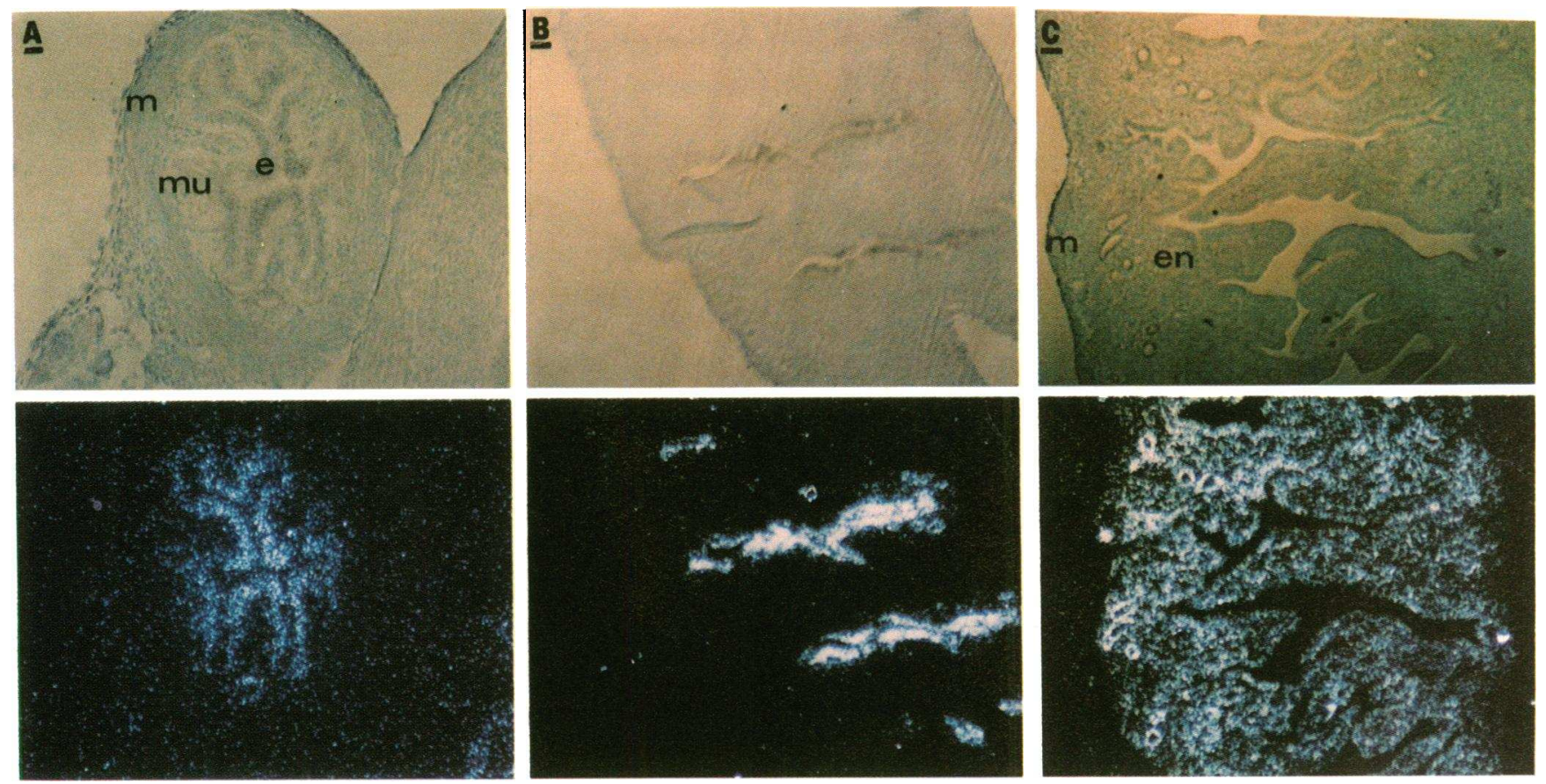

Figure 3. Expression of VEGF in the oviduct and uterus. In situ hybridization with a VEGF-specific probe: $(A)$ a section through the oviduct; $(B$ and $C$ ) sections of the uterus, withdrawn at early proliferating phase and secretory phase, respectively. $m$, muscular layer; $m u$, mucosal layer of the oviduct; $e$, epithelium; en, endometrium.

clearly showing a cycle-dependent pattern of expression of VEGF in the endometrium, suggest that expression of VEGF is hormonally regulated. VEGF produced in the endometrial stroma may serve as a source of angiogenic activity that supports the extension of stromal vessels.

Angiogenesis in the embryonic implantation site. The sequential influence of estrogens and progesterone on the stromal cells of the endometrium makes them capable of undergoing transformation into decidual cells. The stimulus for the transformation is the implantation of the blastocyst. Development of the decidua involves extensive proliferation of decidual cells. Accordingly, an elaborate network of blood vessels is formed within the decidua. At 7-9 d postcoitum, angiogenesis is ongoing and is visible as multiple endothelial cell cords traversing the decidua. The anastomosing network formed converges on the embryo. This spatial arrangement of the vasculature reflects the fact that, later on, the decidua basalis will develop into the maternal contribution of the placenta (see Fig. 4 $C$ for a schematic illustration of the implantation site of a 9.5-d embryo). The unique spatial arrangement of the decidual vascular network is readily visualized through in situ hybridization with the endothelial cell-specific probe von Willebrand factor cDNA (Fig. $4 \mathrm{D}$ ).

To determine whether VEGF may play a role in formation of the unique vascular network of the decidua, we analyzed implantation sites of mouse embryos at $9.5 \mathrm{~d}$ postcoitum with respect to VEGF and VEGF receptor expression. Our findings suggest the following scenario: decidual transformation leads to the upregulation of VEGF expression in all decidual cells (Fig. $4 \mathrm{~A}$ ). Note that the deepest layers of the endometrium (the so-called stratum basale) remains nonexpressing. This is consistent with the fact that decidual transformation includes all but the deepest layer of the endometrium. Moreover, we show that upregulation of VEGF expression takes place concurrently with decidual transformation. To show this point, we made use of a pseudopregnancy model system in which decidual transformation was focally induced through application of oil to the hormonally conditioned, mechanically stimulated uterus (see Methods for details). As shown in Fig. 4 B. VEGF mRNA is only detectable in cells that have undergone decidual transformation, while the bulk of nontransformed uterine cells remained nonexpressing (decidual cells are distinguishable by their large size relative to nontransformed endometrial cells, and are arranged in clusters around the presumed locations of oil droplets). VEGF produced by the decidua may potentially support the proliferation of interspersed endothelial cells, essential for the expansion of the decidual vasculature. To account for the directionality of the vascular network, however, one has to evoke a nonhomogenous distribution of VEGF (or, alternatively, the involvement of additional angiogenic factors). Therefore, we examined whether VEGF is produced in additional cell types within the implantation site. Indeed, we found that highest steady state levels of VEGF mRNA are produced in the extra-embryonic giant trophoblasts cells (Fig. 4 $F$ ). VEGF vastly produced, and presumably secreted by giant trophoblast cells surrounding the embryo, is likely to form a gradient of angiogenic activity directing the growth and/or migration of endothelial cells towards the embryo. Consistent with this assignment, we show that cords of endothelial cells targeted to this site display a potent VEGF-binding activity (Fig. $4 E$ ). Noteworthy, the syncytiotrophoblast is the site of synthesis of both steroids and peptide hormones.

Expression of VEGF in other steroidogenic cells. Strikingly, all steroidogenic cell types included in the in situ survey described above were found to express VEGF mRNA. Therefore, we wished to determine whether the property of VEGF expres- 


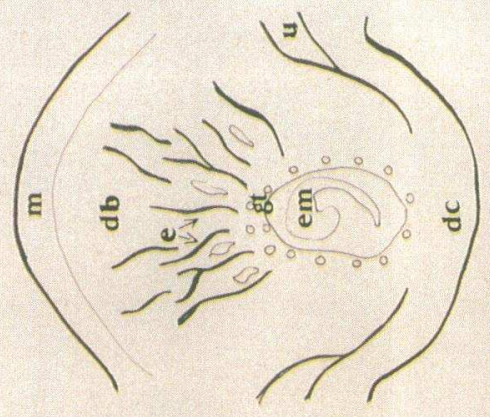

UI
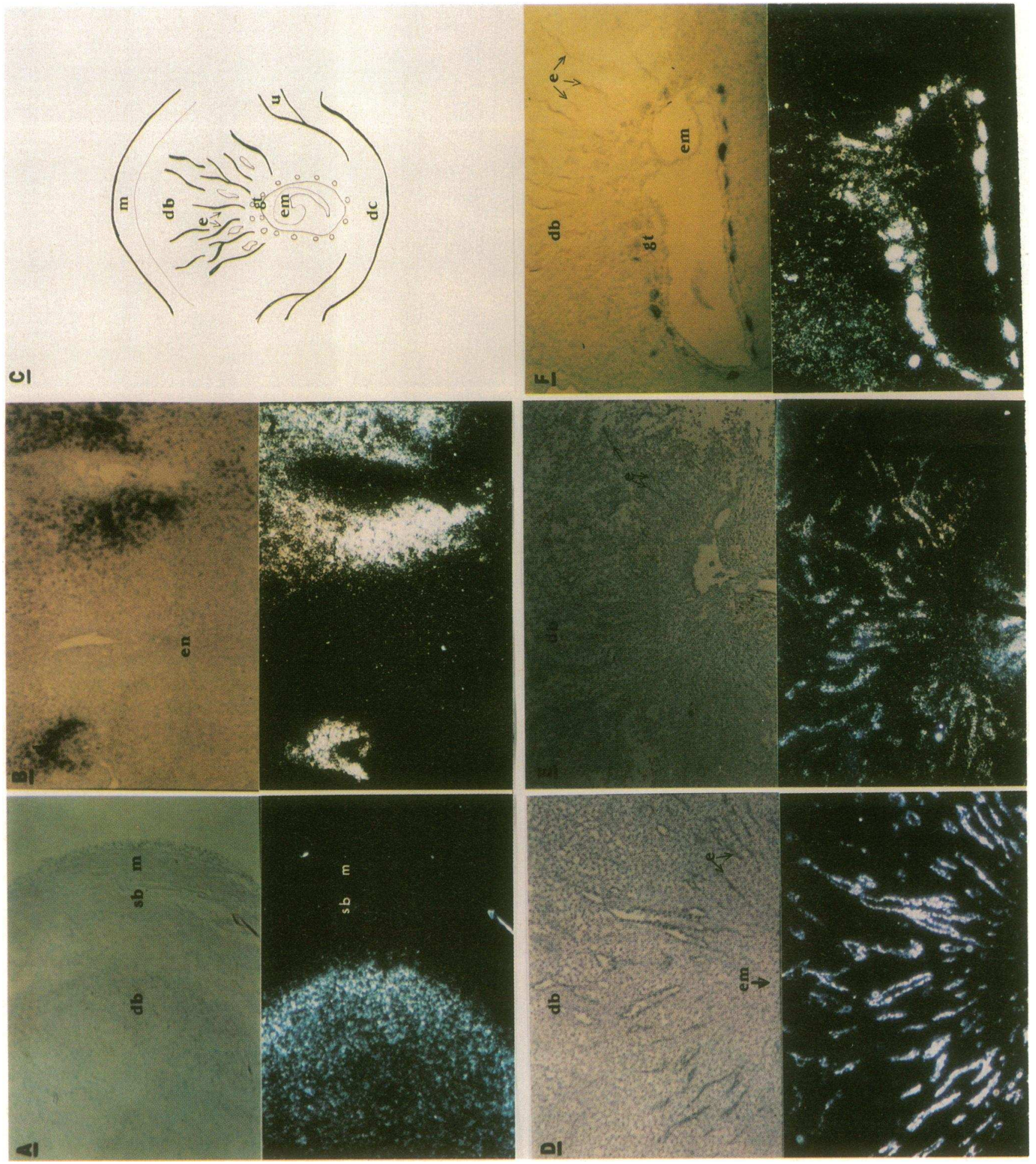
sion is shared by other steroidogenic cell types. In addition to the ovary and placenta, two major steroidogenic organs are the adrenal gland and the testes.

In the adrenal gland, steroidogenesis is confined to the cortex where, under the influence of $\mathrm{ACTH}$, different cortical zones specialize in the synthesis of different steroids. As shown in Fig. $5 A$, VEGF mRNA is abundantly expressed in all cells of the adrenal cortex. The adrenal medulla, which produces nonsteroid hormones, does not express VEGF.

In the testes, specialized interstitial cells termed Leydig cells, elaborate the male steroid hormone testosterone. As shown in Fig. $5 B$, VEGF expression in the testis is confined to Leydig cells, seen scattered in the intterstices among the seminefrous tubules. For comparison, no expression was detected in the tubules.

In conclusion, it appears that VEGF is efficiently expressed in all the steroidogenic cell types examined.

\section{Discussion}

The search for angiogenic factors has yielded a considerable number of factors that elicit an angiogenic response in model systems and/or factors that fulfill certain criteria of angiogenic factors (e.g., endothelial cell mitogens or chemoattractants). The apparent redundancy in putative angiogenic factors has been previously discussed, and the possibilities that redundancy reflects either artifactual promiscuity of the assay systems, or indirect effects of some angiogenic factors, or a situation where different tissues elaborate different angiogenic factors, has been considered (e.g., see reference 27). Yet, the issue of physiological relevance of each candidate factor to any given natural process remains a key unresolved issue in angiogenesis research. Ideally, this issue should be addressed through analysis of natural angiogenesis. Best suitable are programmed processes, where specimens that represent all stages of ongoing angiogenesis, including initiating events and downstream responses, can be retrieved for in situ analysis. The objective of the present study was to analyze in situ angiogenic processes taking place in the reproductive system, with the aim of identifying an angiogenic factor that is expressed in close spatiotemporal proximity to the forming vasculature.

The female reproductive system undergoes a number of programmed neovascularization processes coupled with the cyclic evolution and decline of ovarian and uterine structures. Cycling in the ovary, in general, is initiated through the cyclic secretion of gonadotropins from extraovarian tissues. Functional compartmentalization within the ovary, with respect to gonadotropin receptors and steroidogenic capabilities generates, in turn, a wavelike steroidogenesis within the ovary. Ovarian steroids also govern endometrial cycles. It is most likely, therefore, that the angiogenic waves within the reproductive system are coordinated by gonadotropins and/or by locally produced steroids. This requires that the expression of the initiating angiogenic factor will be hormone responsive (although hormone responsiveness may be indirect). We have examined successive stages in four independent angiogenic processes taking place in the reproductive system. Our findings can be summarized by generalizing that, in all four processes, VEGF is indeed expressed in spatial and temporal proximity to the forming vasculature. Specifically, during follicular neovascularization, VEGF was produced by interstitial and peripheral theca layers; during formation of the $\mathrm{CL}$ vasculature, VEGF production was predominantly detected in lutein cells; during neovascularization of the endometrium, VEGF was detected throughout the endometrial stroma; and during vascularization of the decidua, VEGF was expressed in the decidua basalis. These sites of VEGF expression colocalize with the sites of residence of the new vasculature. VEGF receptors were primarily detected on endothelial cells (e.g., Figs. 2 and $4 E$ ). It should be noted, however, that the limited sensitivity of the in situ receptor-ligand binding assay may have precluded detection of VEGF receptors on additional cell types (28). These results suggest that, during these processes, VEGF acts primarily on nearby endothelial cells. Since VEGF receptors are expressed both in endothelial cells participating in neovascularization, as well as in quiescent endothelium (reference 26 and our unpublished data), it appears that the locally released ligand determines the area of endothelial cell growth by creating a microenvironment that support the proliferation of nearby endothelial cells. Furthermore, uneven distribution of extracellular VEGF in the neovascularized tissue may target capillary growth towards cells producing highest levels of the angiogenic growth factor. For example, we suggest that the massive production of VEGF by the syncytiotrophoblast plays an important role in targeting the decidual network towards the implanted embryo (Fig. 4). Although circumstantial in nature, we believe that our findings support the thesis that VEGF mediates hormonally regulated angiogenesis.

It should be noted that in the systems analyzed, VEGF was also expressed at times where neovascularization was not ongoing; (e.g., in theca and lutein cells after completion of angiogenesis). Thus, VEGF may play additional roles in these systems, possibly in the maintenance of the vasculature, or in controlling its permeability. A similar observation, namely, expression of VEGF during embryonic angiogenesis, but persistence of VEGF expression in the fully developed organ was made by Brier et al. (29) in the case of brain and kidney development.

What mechanism( $s$ ) account for the activation of VEGF in each of the different cell types? And, more specifically, can the activation of VEGF in the respective cell types be accounted

Figure 4. Expression of VEGF and VEGF receptors in the decidua and embryonic implantation site. $(A)$ Decidual tissue of a 9.5-d embryo. Hybridization with a VEGF-specific probe. $(B)$ Uterus of a pseudopregnant female mouse hybridized with a VEGF-specific probe. Note that only a fraction of endometrial cells have undergone decidual transformation. Hybridization with a VEGF-specific probe. $(C)$ A schematic view of an implantation site at 9.5 -d postcoitum. $(D)$ The forming vascular network visualized through in situ hybridization with an endothelial cell-specific von Willebrand factor cDNA probe. Note the orientation of endothelial cell cords traversing the decidua basalis and converging on the embryo. ( $E$ ) Specific binding of ${ }^{125}$ I-labeled VEGF to endothelial cell cords. The region of the implantation site shown is the same as in $D$. $(F)$ In situ hybridization with a VEGF-specific probe. The section shown includes the embryo, extraembryonic tissues, and a portion of the decidua undergoing neovascularization. $m$, myometrium; $s b$, stratum basale; $d b$, decidua basalis; $e n$, endometrial cells; $d$, decidual cells; $e$, endothelial cells; $g t$, giant trohoblast cells; $e m$, embryo; $d c$, decidua capsularis; $u$, uterine lumen. 

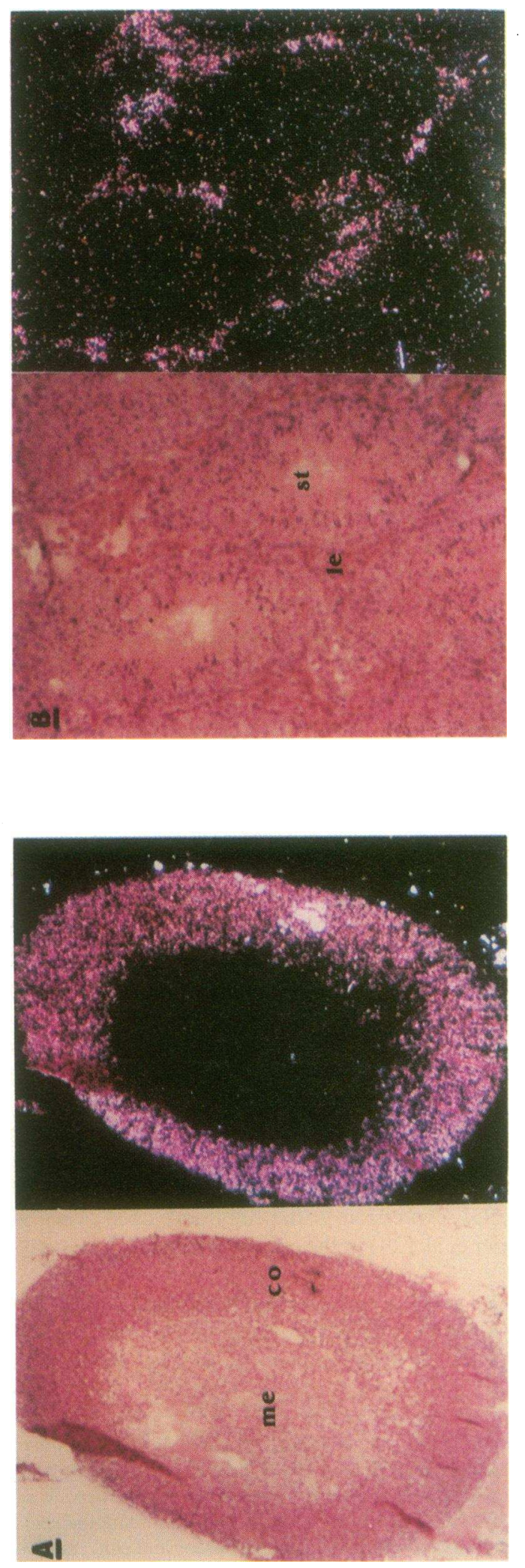

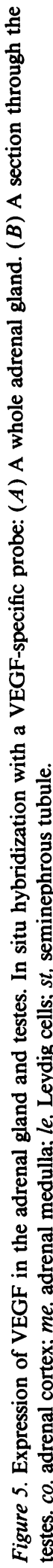

for by hormonal regulation? Together, this in situ study shows the in vivo expression of VEGF in 10 different cell types: theca, cumulus, granulosa, lutein, oviductal and uterine epithelium, endometrial stroma, decidua basalis, syncytiotrophoblast, adrenal cortex, and Leydig cells. Noteworthy, in certain cell types (e.g., granulosa and endometrial stroma) expression of VEGF is temporally restricted, detectable only at some stages of the ovarian or endometrial cycle but not at others. These findings suggest that, at least in these cells, expression of VEGF is hormonally regulated, rather than merely reflecting cell type specificity. All 10 expressing cell types are either steroid hormoneresponsive or steroid hormone producers, or both. Excluding the estrogen-responsive oviductal and endometrial epithelium, all VEGF-expressing cells produce steroids in response to the respective trophic hormone. It is thus possible that VEGF is luteinizing hormone ( $\mathrm{LH})$ - or ACTH- inducible in a similar manner that $\mathrm{LH}$ induces the transcription of $\mathrm{mRNAs}$ encoding steroidogenic enzymes. Consistent with this hypothesis, we find a temporal correlation and colocalization of VEGF mRNA and mRNA of the key steroidogenic enzyme cytochrome P450 side-chain cleavage in the theca, cumulus and CL compartments of the ovary (data not shown). Also, we find that when partial decidualization is induced in the pseudopregnancy model, VEGF expression co-localize with cells expressing P450 side-chain cleavage mRNA (Schiff, R., Y. Arensburg, A. Itin, E. Keshet, and J. Orly, manuscript in preparation). Alternatively, expression of VEGF might be steroid-inducible. In this case, expression in steroidogenic cells might be induced by steroids acting autocrinically. Obviously, different mechanisms of activation might be operating in the different cell types shown here to express VEGF. In any event, the study reported here provides a suitable in vivo framework for further in vitro studies addressing the issue of hormonal regulation of VEGF expression.

Certain steroids are known to possess angiostatic activity (30). Yet, the molecular basis for the antiangiogenic activity of steroids is poorly understood. We hope that further analysis of natural, self-limiting angiogenic processes, in which both the "on" and "off" switches are likely to be regulated by hormones, will shed light on this issue. Finally, VEGF is known to possess a potent vascular permeabilization activity $(31,32)$. Thus, overstimulation of VEGF expression might lead to excessive vascular permeability. The ovarian hyperstimulation protocol used in these experiments, leading to high levels of VEGF expression in a large number of follicles and corpora lutea, is similar to the one used in ovulation induction therapy, where the recruitment and maintenance of a large number of ovarian follicles is induced by gonadotropins follicle-stimulating hormone combined with an unphysiological dose of human chorionic gonadotropin ( $\mathrm{LH})$. A main complication of ovulation induction therapy is the ovarian hyperstimulation syndrome (OHSS). Interestingly, the underlying problem in OHSS is the shift of body fluids from the vascular bed into the extravascular space. We speculate that excessive production of VEGF might cause or at least contribute to OHSS. This proposal is currently under investigation.

\section{Acknowledgments}

This work was supported by grants from the Charles H. Revson Fund, (administered by the Israeli Academy of Sciences) and from the Israeli 
Ministry for Health (to E. Keshet) and by a grant from the USA-Israel Binational Fund (to G. Neufeld).

\section{References}

1. Gospodarowicz, D., and K. K. Thakral. 1978. Production of corpus luteum angiogenic factor responsible for proliferation of capillaries and neovascularization of the corpus luteum. Proc. Natl. Acad. Sci. USA. 75:847-851.

2. Koos, R. D., and W. J. Le Maire. 1983. Factors that may regulate the growth and regression of blood vessels in the ovary. Semin. Reprod. Endocrinol. 1:295-307.

3. Findlay, J. K. 1986. Angiogenesis in reproductive tissues. J. Endocrinol. 111:357-366.

4. Christianes, G. C. M. L., J. J. Sixma, and A. A. Haspels. 1982. Hemostasis in menstrual endometrium: a review. Obstet. Gynecol. Survey. 37:281-303.

5. Motro, B., A. Itin, L. Sachs, and E. Keshet. 1990. Pattern of interleukin 6 expression in vivo suggests a role in angiogenesis. Proc. Natl. Acad. Sci. USA. 87:3092-3096.

6. Folkman, J., and M. Klagsbrun. 1987. Angiogenic factors. Science (Wash DC). 235:442-447.

7. Risau, W. 1990. Angiogenic growth factors. Progress in growth factors research. Neuroendocrinol. Immunol. 2:71-79.

8. Klagsbrun, M., and P. A. D’Amore. 1991. Regulators of angiogenesis. Annu. Rev. Physiol. 53:217-239.

9. Bassett, D. L., 1943. The changes in the vascular pattern of the ovary of the albino rat during the esrous cycle. Am. J. Anat. 73:251-291.

10. Ferrara, N., K. Houck, L. Jakeman, and D. W. Leung. 1992. Molecular and biological properties of the vascular endothelial growth factor family of proteins. Endocr. Rev. 13:18-32.

11. Connoly, D. T., D. M. Heuvelman, R. Nelson, J. V. Olander, B. L. Eppley, J. J. Delfino, N. R. Siegel, R. M. Leimgruber, and J. Feder. 1989. Tumor vascular permeability factor stimulates endothelial cell growth and angiogenesis. J. Clin. Invest. 84:1470-1478.

12. Leung, D. W., G. Cachianes, W.-J. Kaung, D. V. Goeddel, and N. Ferrara 1989. Vascular endothelial growth factor is a secreted angiogenic mitogen. Science (Wash. DC). 246:1306-1309.

13. Plouet, J., J. Schilling, and D. Gospodarowitz. 1989. Isolation and characterization of a newly identified endothelial cell mitogen produced by AtT-20 cells. EMBO (Eur. Mol. Biol. Organ.) J. 8:3801-3806.

14. Clauss, M., H. Gerlach, J. Brett, F. Wang, P. C. Familletti, Y.-C. E. Pan, J. V. Olander, D. T. Connolly, and D. Stern. 1990. Vascular permeability factor: A tumor-derived polypeptide that induces endothelial cell and monocyte procoagulant activity, and promotes monocyte migration. J. Exp. Med. 172:1535-1545.

15. Gospodarowitz, D., J. A. Abraham, and J. Schilling. 1989. Isolation and characterization of vascular endothelial mitogen produced by pituitary-derived folliculo stellate cells. Proc. Natl. Acad. Sci. USA. 86:7311-7315.

16. Conn, G., D. D. Soderman, M.-T. Scaeffer, M. Wile, V. Hatcher, and
K. A. Thomas. 1990. Purification of a glycoprotein vascular endothelial cell mitogen from a rat glioma-derived cell line. Proc. Natl. Acad. Sci. USA. 87:13231327.

17. Phillips, H. S., J. Hains, D. W. Leung, and N. Ferrara. 1990. Vascular endothelial growth factor is expressed in rat corpus luteum. Endocrinology 127:965-967.

18. Finn, C. A. 1965. Oestrogen and the decidual cell reaction of implantation in mice. $J$. Endocrinol. 32:223-229.

19. Hogan, B., F. Constantini, and E. Lacy. 1986. Manipulating the Mouse Embryo: A Laboratory Manual. Cold Spring Harbor Laboratory Press, Cold Spring Harbor, NY. 219-244.

20. Bonthron, D., E. C. Orr, L. M. Mitsock, D. Ginsburg, R. I. Handin, and S. H. Orkin. 1986. Nucleotide sequence of pre-pro-von Willebrand factor cDNA. Nucleic Acids Res. 14:7125-7127.

21. Cohen, Z., H. Gitay-Goren, G. Neufeld, and B.-Z. Levi. 1992. High levels of biologically active vascular endothelial growth factor are produced by the Baculovirus expression system. Growth Factors. 7:131-138.

22. Vaismann, N., D. Gospodarowicz, and G. Neufeld. 1990. Characterization of the receptor for vascular endothelial growth factor. Biol. Chem. 265:19461-19466

23. Marchetti, B., M. C. Morale, and G. Pelletier. 1990. Sympathetic nervous system control of rat thymus gland maturation: autoradiographic localization of the b2-adrenergic receptor in the thymus and presence of sexual dimorphism during ontogeny. Prog. in Neuroendocrinol. Immunol. 3:103-115.

24. Dvorak, H. F., T. M. Sioussat, L. F. Brown, B. Berse, J. A. Nagy, A. Sortel, J. Manseau, L. Van De Water, and D. R. Senger. 1991. Distribution of vascular permeability factor ( vascular endothelial growth factor) in tumors: concentration in tumor blood vessels. J. Exp. Med. 174:1275-1278.

25. Gates, A. H. 1971. Maximizing yield and developmental uniformity of eggs. In Methods in Mammalian Embryology. J. C. Daniel and W. H. Freeman, editors. San Francisco, CA. 46-76.

26. Jakeman, L. B., J. Winer, G. L. Bennett, C. A. Altar, and N. Ferrara. 1992. Binding sites for vascular endothelial growth factor are localized on endothelial cells in adult rat tissues. J. Clin. Invest. 89:244-253.

27. Zetter, B. R. 1988. Angiogenesis, state of the art. Chest. 93:159S-166S.

28. Gitay-Goren, H., S. Sofer, I. Vlodavsky, and G. Neufeld. 1992. The binding of vascular endothelial growth factor to its receptor is dependent on cell-surface associated heparin-like molecules. J. Biol. Chem. 267:6093-6098.

29. Brier, G., U. Albrecht, S. Sterrer, and W. Risau. 1992. Expression of vascular endothelial growth factor during embryonic angiogenesis and endothelial cell differentiation. Development. 114:521-532.

30. Folkman, J., and D. E. Ingber. 1987. Angiostatic steroids: method of discovery and mechanism of action. Ann. Surg. 206:374-384.

31. Senger, D. R., S. J. Galli, A. M. Dvorak, C. A. Perruzzi, V. S. Harvey, and H. F. Dvorak. 1983. Tumor cells secrete a vascular permeability factor that promotes accumulation of ascites fluid. Science (Wash. DC). 219:983-985.

32. Keck, P. J., S. D. Hauser, G. Krivi, K. Sanzo, T. Warren, J. Feder, and D. T. Connolly. 1989. Vascular permeability factor, an endothelial cell mitogen related to PDGF. Science (Wash. DC). 246:1309-1312. 